\title{
Cystic lymphoid hyperplasia of the parotid gland as the initial manifestation of HIV infection
}

\author{
Bingcheng $W u^{1}$, MBBS, Raymond $\underline{N g o}^{2}$, MBBCh BA, FAMs, Fredrik Petersson ${ }^{1}, \mathrm{MD}, \mathrm{PhD}$
}

\begin{abstract}
We report the case of a patient who presented with cystic lymphoid hyperplasia of the right parotid gland as the index diagnosis of HIV infection. Histological examination of the excised parotid gland revealed a solid-cystic lymphoepithelial lesion with a non-keratinous squamous epithelium, which grew into the lymphoid component via anastomosing cords and islands. These anastomosing cords and islands contained variably abundant B cells, several subepithelial multinucleated histiocytes, salivary ducts infiltrated by small lymphocytes, and a dense lymphoid infiltrate containing lymphoid follicles with enlarged, irregular germinal centres.
\end{abstract}

Keywords: AIDS, HIV-associated salivary gland disease, human immunodeficiency virus, lymphoepithelial sialadenitis, Sjögren's syndrome

\section{INTRODUCTION}

It is reported that $3 \%-10 \%$ of patients (both adults and children) infected with the human immunodeficiency virus (HIV) develop lesions in the salivary glands. An overwhelming majority of these are cystic lymphoepithelial lesions (cLELs). ${ }^{(1-3)}$ These salivary gland lesions commonly precede the development of acquired immunodeficiency syndrome (AIDS), but may be the initial clinical manifestation of HIV infection. ${ }^{(4)}$ HIV-associated cystic lymphoepithelial salivary gland disease (H-SGD) is typified by florid lymphoid hyperplasia in conjunction with an epithelial component that displays lymphotropism, cystic and proliferative changes, and the formation of lymphoepithelial islands/complexes. The histomorphological and cytopathological features of HIVassociated CLELs overlap with several benign and malignant entities that have radically different prognoses and approaches to treatment. We herein report a patient who presented with a benign CLEL, and was found to be infected with HIV. We also discuss the differential diagnostic aspects of salivary gland lesions that are composed of lymphoid and epithelial components.

\section{CASE REPORT}

A previously healthy 42-year-old man presented with a one-year history of a right parotid lump. There was no cervical lymphadenopathy or facial nerve palsy. Computed tomography (CT) showed a well-defined, 2.4-cm cystic lesion located in the right parotid gland (Fig. 1).

A $1.3-\mathrm{cm}$ cystic lesion located in a similar position in the contralateral side of the patient's neck was also identified. The right-sided cystic parotid mass was subjected to fine needle aspiration (FNA) biopsy. The biopsy specimen yielded scattered macrophages and a few small lymphocytes, and was interpreted as cyst fluid. The patient subsequently underwent a right

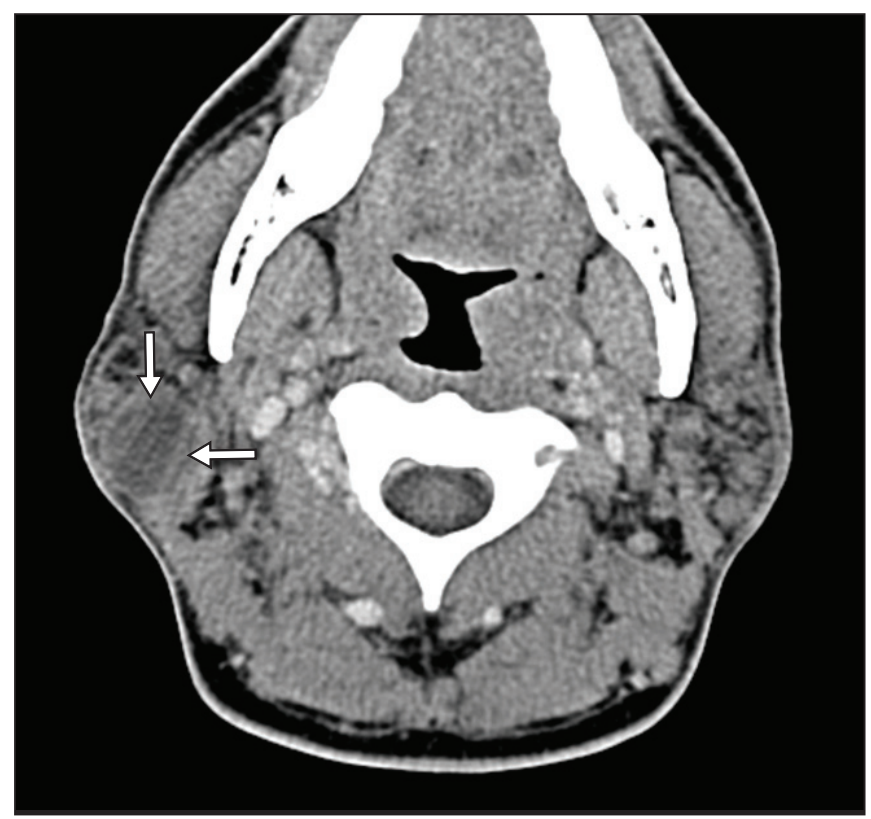

Fig. 1 CT image of the neck shows a well-defined cystic lesion (arrows) in the right parotid gland.

superficial parotidectomy. Intraoperatively, a well-circumscribed parotid lesion measuring $6.0 \mathrm{~cm} \times 3.0 \mathrm{~cm} \times 2.5 \mathrm{~cm}$ was identified. After histopathological examination of the resected lesion, the patient underwent serological testing for HIV. The patient was found to be positive for HIV, with an HIV-1 viral load of 78,800 copies $/ \mathrm{mL}$. The patient had an absolute CD4 count of 295 cells/uL and a CD4/CD8 ratio of 0.15 . The patient was not started on highly active antiretroviral therapy at the time of diagnosis, but was administered co-trimoxazole prophylaxis.

Histological examination on low power magnification revealed a multinodular, solid-cystic lesion composed of a dense lymphoid infiltrate, featuring multiple lymphoid follicles

${ }^{1}$ Department of Pathology, ${ }^{2}$ Department of Otolaryngology, National University Health System, Singapore

Correspondence: Dr Fredrik Petersson, Associate Professor and Senior Consultant, Department of Pathology, National University Health System, 5 Lower Kent Ridge Road, Singapore 119074. fredrikpetersson@live.se 

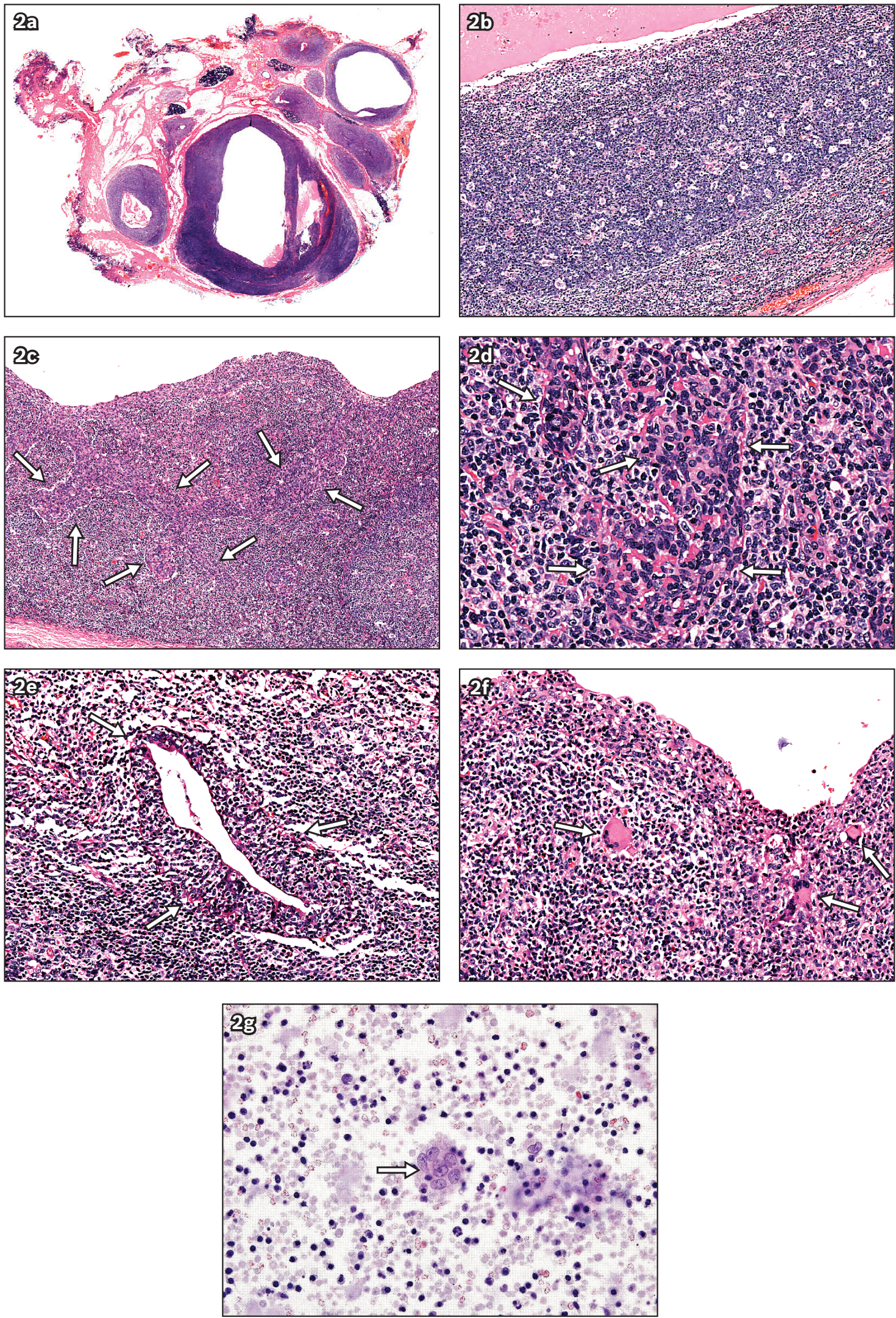

Fig. 2 Photomicrographs of the HIV-associated lymphoepithelial parotid lesion show: (a) a multinodular solid-cystic lesion (Haematoxylin \& eosin, low power magnification); (b) enlarged, irregular germinal centres containing numerous tingible body macrophages in the lymphoid component (Haematoxylin \& eosin, medium to high power magnification); (c) broad anastomosing cords of squamous epithelium (arrows) in the lymphoid component (Haematoxylin \& eosin, medium power magnification); (d) lymphoepithelial islands/complexes with variable amounts of hyaline (basement membrane-like) material (arrows) (Haematoxylin \& eosin, high power magnification); (e) a dilated duct (arrows) infiltrated by small lymphocytes (Haematoxylin \& eosin, medium to high power magnification); (f) multinucleated giant cells (arrows) close to the cystic squamous epithelium (Haematoxylin \& eosin, medium to high power magnification); and (g) an occasional multinucleated histiocyte (arrow), which was identified when the preoperative fine needle aspiration cytology was reviewed (Haematoxylin \& eosin, high power magnification). 
with enlarged, irregular germinal centres containing tingible body macrophages (Figs. 2a \& b). The epithelium of the cyst was composed of non-keratinous squamous cells overlying a dense lymphoid infiltrate. In several areas, the squamous epithelium grew down into the subjacent lymphoid component, forming anastomosing broad cords (Fig. 2c) and basaloid epithelial islands with variably abundant intraepithelial lymphocytes, i.e. Iymphoepithelial islands. Some of these lymphoepithelial islands harboured eosinophilic basement membrane-like material (Fig. 2d). In addition, there were several variably dilated ducts where the epithelium was infiltrated by small lymphoid cells with uniform round nuclei and a moderate amount of pale cytoplasm (Fig. 2e). There were several foci containing small clusters of multinucleated giant cells; these foci were interspersed within the interfollicular regions and most were situated close to the cystic squamous epithelial component (Fig. 2f). The background inflammatory cell population also contained macrophages, plasma cells and a few neutrophils. The salivary gland tissue outside the main lesion displayed similar changes as described above, albeit patchy and less prominent. No well-formed granulomas, sheets of monocytoid B cells or plasma cells were identified.

The FNA smears were reviewed, and in addition to the small lymphoid cells and macrophages seen at the initial microscopic examination, we identified occasional multinucleated histiocytes (Fig. 2g). Immunohistochemical study showed that the lymphoid component was composed of CD20 positive $B$ cells, and CD3- and CD5-positive T cells. The T cells were mainly identified in the interfollicular regions, but were also found scattered within the lymphoid follicles. The germinal centre B cells expressed CD10, but not Bcl2. CD21 revealed preserved and focally expanded follicular dendritic meshworks. The lymphoid cells located within the epithelium of the cyst and epithelial islands were predominantly CD20positive B cells, with a few scattered CD3-positive T cells. No co-expression of CD43 and PAX5 was identified. No light chain restriction or immunoglobulin heavy chain clonal rearrangement was detected. Immunohistochemistry for broad-spectrum cytokeratins (AE1/3) highlighted the epithelial component.

\section{DISCUSSION}

A minor but significant proportion $(3 \%-10 \%)$ of patients with HIV infection develops lesions in the salivary glands. Most of these salivary gland lesions display cystic and lymphoepithelial features on histopathological examination. ${ }^{(1-3)}$ Although the diagnosis of H-SGD most commonly precedes AIDS, these salivary gland lesions may be the initial clinical manifestation of HIV infection. ${ }^{(4)}$ HIV-associated cLELs are typified by florid lymphoid hyperplasia in conjunction with an epithelial component that displays lymphotropism, as well as cystic and proliferative changes, together with the formation of lymphoepithelial islands.
Clinically, H-SGD most often presents with bilateral salivary gland enlargement and/or xerostomia.(5) Imaging studies often identify the presence of multiple parotid 'cysts'. ${ }^{(6)}$ H-SGD frequently regresses upon antiretroviral therapy, and its incidence has significantly decreased in areas of the world where HIVinfected patients have good access to antiviral therapies. ${ }^{(5,7)}$ Although a majority of the lesional cells found in H-SGD do not harbour the virus, exuberant lymphoid hyperplasia and associated proliferative epithelial changes may be related to the viral load, thus explaining the regression of the disease with the administration of antiviral therapy. ${ }^{(3)}$ The histomorphologic features of H-SGD include dense lymphoid infiltration of the salivary gland with acinar destruction and formation of lymphoepithelial complexes/islands, as well as development of lymphoepithelial cysts. There are numerous lymphoid follicles within the lymphoid infiltrate. Many of these lymphoid follicles are large and irregularly shaped with attenuated mantle zones, and may show variable lysis of the germinal centres. In addition to lymphoid cells, the interfollicular lymphoid tissue contains a mixed inflammatory infiltrate, including histiocytes, neutrophils and plasma cells. The majority of the lymphoid cells in the lymphoepithelial islands/complexes are CD20-positive B cells, while most interfollicular lymphocytes are CD8positive T cells. ${ }^{(8)}$

Salivary gland lesions composed of both epithelial (with variable degrees of cystic change) and lymphoid components have a wide-ranging differential diagnosis, which includes several entities with different aetiopathogeneses, treatment approaches and prognoses. Although non-HIV-associated cLELs show significant histopathological overlap with their HIV-associated counterpart, the presence of lymphoepithelial complexes and multinucleated histiocytes are more commonly encountered in HIV-associated cLELs. However, the former has been reported also to occur in non-HIV associated CLELs. ${ }^{(9)}$ Clinically, HIV-associated cLELs tend to occur as bilateral parotid masses; unilateral involvement is more commonly found in sporadic non-HIV-related cLELs. ${ }^{(10)}$

Based on histomorphology alone, the most difficult differential diagnosis of H-SGD is lymphoepithelial sialadenitis (LESA). LESA is an autoimmune exocrinopathy that often occurs in the context of Sjögren's syndrome, which predominantly affects middle-aged or older women. The histopathologic features of LESA are similar to those of H-SGD, though cystic components are more commonly found in H-SGD. However, their absence or presence does not reliably distinguish H-SGD from LESA. ${ }^{(8)}$ In contrast, the presence of aggregates of multinucleated histiocytes, frequently in juxtaposition to the epithelium of the cyst, is characteristic of H-SGD, thus strongly suggesting this possibility rather than LESA. Although the salivary glands are affected in 90\% of cases of Sjögren's syndrome and bilateral involvement is the rule, this bilateral involvement may be asymmetrical..$^{(8)}$ One difference between patients with Sjögren's syndrome-associated LESA and those 
with H-SGD is that the former group of patients displays involvement of the minor salivary glands of the oral mucosa. Moreover, serologically, patients with Sjögren's syndrome exhibit autoantibodies against SSA/Ro and SSB/La.

Given the fact that most, if not all, cases of extranodal marginal zone B cell lymphoma (EMZBCL) of salivary glands arise from pre-existing LESA, coupled with the histopathological overlap between LESA and H-SGD, the differential diagnosis may be difficult. EMZBCL is the most common lymphoma affecting salivary glands, accounting for approximately half of all salivary gland lymphoma cases. The earliest stage of transition of LESA to EMZBCL is characterised by an expansion of monocytoid and/or centrocyte-like B cells around the lymphoepithelial complexes. Although the early stages of transformation of LESA to EMZBCL are subtle, a low-power view of marginal zone $B$ cells forming halos around the LELs may help in rendering the diagnosis. The neoplastic lymphoid B cells subsequently expand and become confluent, forming interconnected sheets and colonising the marginal zones. Immunohistochemically, these neoplastic B cells are typically positive for CD20 and CD79a, and negative for CD5, CD10, and CD23. ${ }^{(8)}$ The co-expression of B cell markers (e.g. Pax-5 and CD43 proteins) is common and strongly supports the neoplastic nature of the cells.

Although H-SGD usually presents with bilateral parotid disease, this process may present unilaterally. In such instances, an epithelial neoplasm with an associated lymphoid component has to be included in the differential diagnosis. These neoplasms mainly include lymphadenomas (LAs) and lymphadenocarcinomas, with or without sebaceous differentiation. LAs are well-circumscribed tumours composed of cytologically bland neoplastic epithelial cells arranged in islands and duct-like structures, occasionally forming cysts within a dense lymphoid stroma. Although these epithelial cells most commonly display squamoid features, the cystic component, when present, may show cuboidal or columnar morphology. Importantly, unlike in LESA, H-SGD, EMZBCL and lymphoepithelial carcinoma, intraepithelial lymphocytic infiltration is not usually evident. ${ }^{(8)}$ Lymphadenocarcinoma, sebaceous or otherwise, exhibits varying degrees of nuclear atypia, increased mitotic activity, and most importantly, infiltrative growth.

Primary lymphoepithelial carcinoma (LECA) of the salivary glands is biologically and histologically analogous to nonkeratinising nasopharyngeal carcinoma. LECA has a peculiar and hitherto unexplained skewed geographical distribution. This tumour is several hundred times more common among Inuits living in the arctic regions (where LECA is the most common salivary gland malignancy), and is also significantly more common among the Chinese, especially those living in Southeast Asia. These tumour cells display malignant cytomorphological features (e.g. nuclear enlargement, pleomorphism and high nuclear to cytoplasmic ratio) and are distributed in a variety of arrangements (e.g. irregular nests, cords, trabeculae, syncytial sheets and isolated cells). A dense, reactive lymphoplasmacytic infiltrate accompanies the malignant epithelial cells, and the lymphoid component frequently contains a varying number of lymphoid follicles. The reactive lymphoid cells frequently permeate the nests of carcinomatous cells. This is not uncommon, to such an extent that the recognition of the carcinomatous component can be difficult on morphological examination, requiring immunohistochemical examination with antibodies against cytokeratins, in order to detect the carcinomatous cells. Although infiltrative, LECA may appear as a deceptively circumscribed nodule. There has been a report of a case of primary LECA of the parotid gland arising in an intraparotid lymph node, which, in addition to mimicking a metastasis from a nasopharyngeal primary carcinoma, could have been mistaken for a benign tumour on low-power examination. (11) The nuclear pleomorphism and increased mitotic activity of the malignant epithelial cells are critical clues to establish the correct diagnosis. In situ hybridisation for Epstein-Barr virus (EBV)-encoded RNA 1 is a useful diagnostic tool as tumour cells, especially in endemic areas, are almost invariably infected with EBV.

Chronic sclerosing sialadenitis (CSS) most commonly affects the submandibular gland and is characterised by a predominantly lymphocytic inflammatory infiltrate, progressive fibrosis and parenchymal atrophy. The aetiology of this condition is traditionally viewed as occurring secondary to sialolithiasis. Recently, however, an association with immunoglobulin G4 (IgG4) sclerosing disease has been established (at least in a subset of patients with CSS). ${ }^{(12)}$ Unlike LESA, H-SGD or MZBCL, CSS shows no prominence of lymphoepithelial complexes. The dense fibrosis that characterises fully developed to late stages of CSS is characteristically absent in H-SGD. Immunohistochemical examination and (semi-)quantitative assessment of the ratio of IgG4-positive to total IgG-positive plasma cells, and serum analysis of lgG4 levels are helpful in establishing the correct diagnosis. ${ }^{(12)}$

The aspiration cytology features of H-SGD, which include a heterogeneous lymphoid population, scattered single and/or clustered foamy macrophages, squamous cells and/or anucleate keratin, have been previously reported. ${ }^{(13)}$ The presence of large multinucleated giant cells is another characteristic of H-SGD that would point toward the correct diagnosis. ${ }^{(13)}$ However, as demonstrated in our case, the number of multinucleated giant cells may be sparse (if present at all), making the cytopathological diagnosis challenging. A high degree of diagnostic suspicion and awareness of this entity would facilitate either the arrival at or the suggestion of the correct diagnosis.

\section{REFERENCES}

1. Vargas PA, Mauad T, Böhm GM, Saldiva PH, Almeida OP. Parotid gland involvement in advanced AIDS. Oral Dis 2003; 9:55-61.

2. Ihrler S, Zietz C, Riederer A, Diebold J, Löhrs U. HIV-related parotid lymphoepithelial cysts. Immunohistochemistry and 3-D reconstruction of surgical and autopsy material with special reference to formal pathogenesis. Virchows Arch 1996; 429:139-47. 
3. Dave SP, Pernas FG, Roy S. The benign lymphoepithelial cyst and a classification system for lymphocytic parotid gland enlargement in the pediatric HIV population. Laryngoscope 2007; 117:106-13.

4. Craven DE, Duncan RA, Stram JR, et al. Response of lymphoepithelial parotid cysts to antiretroviral treatment in HIV-infected adults. Ann Intern Med 1998; 128:455-9.

5. Schiødt M, Dodd CL, Greenspan D, et al. Natural history of HIV-associated salivary gland disease. Oral Surg Oral Med Oral Pathol 1992; 74:326-31.

6. Ellis GL. Lymphoid lesions of salivary glands: malignant and benign. Med Oral Patol Oral Cir Bucal 2007; 12:E479-85.

7. McArthur CP, Subtil-DeOliveira A, Palmer D, et al. Characteristics of salivary diffuse infiltrative lymphocytosis syndrome in West Africa. Arch Pathol Lab Med 2000; 124:1773-9.

8. Ellis GL. Diagnostic approach to lymphoid lesions of major salivary glands.
Diagn Histopathol 2012; 18:381-7.

9. Kojima M, Nakamura N, Matsuda $\mathrm{H}$, et al. HIV-unrelated benign lymphoepithelial cyst of the parotid glands containing lymphoepithelial lesion-like structures: a report of 3 cases. Int J Surg Pathol 2009; 17:421-5.

10. Greaves WO, Wang SA. Selected topics on lymphoid lesions in the head and neck regions. Head Neck Pathol 2011; 5:41-50.

11. Gupta S, Loh KS, Petersson F. Lymphoepithelial carcinoma of the parotid gland arising in an intraglandular lymph node: report of a rare case mimicking metastasis. Ann Diagn Pathol 2012; 16:416-21.

12. Geyer JT, Ferry JA, Harris NL, et al. Chronic sclerosing sialadenitis (Küttner tumor) is an IgG4-associated disease. Am J Surg Pathol 2010; 34:202-10.

13. Gupta N, Gupta R, Rajwanshi A, Bakshi J. Multinucleated giant cells in HIV-associated benign lymphoepithelial cyst-like lesions of the parotid gland on FNAC. Diagn Cytopathol 2009; 37:203-4. 